\title{
TROPOSPHERIC CORRECTION FOR INSAR USING INTERPOLATED ECMWF DATA AND GPS ZENITH TOTAL DELAY FROM THE SOUTHERN CALIFORNIA INTEGRATED GPS NETWORK
}

\author{
J. S. Löfgren, F. Björndahl \\ Chalmers University of Technology \\ Department of Radio and Space Science \\ Onsala Space Observatory, \\ SE-439 92 Onsala, Sweden
}

\begin{abstract}
A tropospheric correction method for Interferometric Synthetic Aperture Radar (InSAR) was developed using profiles from the European Centre for Medium-Range Weather Forecasts (ECMWF) and Zenith Total Delay (ZTD) from the Global Positioning System (GPS). The ECMWF data were interpolated into a finer grid with the Stretched Boundary Layer Model (SBLM) using a Digital Elevation Model (DEM) with a horizontal resolution of 1 arcsecond. The output were converted into ZTD and combined with the GPS ZTD in order to achieve tropospheric correction maps utilizing both the high spatial resolution of the SBLM and the high accuracy of the GPS. These maps were evaluated for three InSAR images, with short temporal baselines (implying no surface deformation), from Envisat during 2006 on an area stretching northeast from the Los Angeles basin towards Death Valley.

The RMS in the InSAR images was greatly reduced, up to $32 \%$, when using the tropospheric corrections. Two of the residuals showed a constant gradient over the area, suggesting a remaining orbit error. This error was reduced by reprocessing the troposphere corrected InSAR images with the result of an overall RMS reduction of $15-68 \%$.
\end{abstract}

Index Terms - InSAR, tropospheric correction, GPS, zenith total delay, stretched boundary layer model, ECMWF

\section{INTRODUCTION}

Interferometric Synthetic Aperture Radar (InSAR) is a technique used to generate images of surface deformation or elevation. It combines two images from a Synthetic Aperture Radar (SAR) satellite, to enable measurements of the vertical component, and has proven to be a powerful technique for mapping topography changes down to sub-centimeter level [1]. One significant error source in InSAR imaging is the propagation delay of radio signals in the atmosphere [2]. A major contribution to the delay is the highly variable water
A. W. Moore, F. H. Webb, E. J. Fielding, E. F. Fishbein

\author{
Jet Propulsion Laboratory \\ California Institute of Technology \\ 4800 Oak Grove Dr., \\ Pasadena CA, 91109, USA
}

vapor content in the troposphere and it was suggested that a spatial or temporal change in relative humidity of $20 \%$ would lead to $10 \mathrm{~cm}$ of errors in deformation products [3]. The current way to reduce tropospheric effects in InSAR images is to average several independent interferograms. In this statistical method the tropospheric delay is regarded as white noise, implying that there is no correlation between variations in the tropospheric delay and topography. Nevertheless, when comparing the tropospheric delay and the topography it is evident that correlation exists, hence new methods are needed. An alternative approach to reduce tropospheric errors in InSAR images is to use Zenith Total Delay (ZTD) estimated from the Global Positioning System (GPS). Corrections using interpolated ZTD from GPS have shown promising results (e.g. [4]; [5]; [6]). However, the limited spatial coverage of GPS stations raises the need for a method with higher spatial resolution. A way to increase the spatial resolution could be to introduce another dataset and combine this with the GPS ZTD. In this paper, a higher spatial resolution is achieved by using the Stretched Boundary Layer Model (SBLM). This model interpolates forecast data from the European Centre for Medium-Range Weather Forecasts (ECMWF) into a finer resolution grid of Total Precipitable Water Vapor (TPW) using a Digital Elevation Model (DEM). These grids are then converted into ZTD difference maps (tropospheric correction maps). Results are presented from tropospheric correction of three interferograms from Envisat in 2006 using both ZTD difference maps from the SBLM and interpolated GPS ZTD from the Southern California Integrated GPS Network (SCIGN), separately, and from a combination that utilizes both the high spatial resolution of the SBLM and the high accuracy of the GPS ZTD.

\section{SYNTHETIC APERTURE RADAR DATA}

The SAR data were aquired from European Space Agency (ESA) Envisat track 170 during 2006 spanning from the Los Angeles basin in the south to Death Valley in the north, 
defined by the corners $\varphi_{\min }:-118.553^{\circ} \mathrm{W}, \varphi_{\max }:-$ $116.517^{\circ} \mathrm{W}, \lambda_{\min }: 33.084^{\circ} \mathrm{N}$, and $\lambda_{\max }: 36.347^{\circ} \mathrm{N}$. In order to evaluate the results of the tropospheric corrections, InSAR images without any other source of decorrelation than from the atmosphere (in particular, without surface deformation) were desired. Therefore, SAR images with the shortest possible temporal differences were preferred, to exclude deformation. With this in mind, and the fact that the spatial baseline between the two acquisition times has to be short (in order to achieve an acceptable correlation), three SAR image pairs were selected. Two of the pairs had the shortest possible temporal baseline of 35 days (July-August and October-November) and one had a temporal baseline of 105 days (June-September). The time of the overpasses was 18 UT.

The interferograms from the three pairs of Envisat SAR images were processed using the Repeat Orbit Interferometry package (ROI_pac) software version 2.3 [7]. The processing was done with SNAPHU unwrapping [8] and the topographic component of the interferometric phase was removed [9]. In order to remove low correlation data from the resulting InSAR images, the coherence image for each pair was used as a mask. For every pixel with correlation less than 0.3 , the corresponding pixel in the InSAR image was removed.

\section{ZENITH TOTAL DELAY FROM GPS}

The GPS data were provided by the SCIGN. Data from all of the available stations located inside the Envisat track were selected for the specified days and time. Additionally, data from adjacent stations outside the area were also selected. This was done in order to mitigate edge effects when later interpolating the data. The number of stations used was between 132 and 135 stations. The distribution of the stations was sparse in the northern parts of the Envisat track, whereas the stations in the south were more evenly distributed with a large cluster in the southwest.

The GPS data were processed separately for each day estimating ZTD using the GIPSY-OASIS II software [10] in Precise Point Positioning (PPP) mode [11] with JPL's Flinn final precise orbit solution. A $7^{\circ}$ elevation cutoff was applied together with the Global Mapping Function [12].

The resulting ZTD data for each station were then interpolated into ZTD maps for each day using Inverse Distance Weighted (IDW) interpolation (depending on the square of the distance) and Gaussian interpolation (with full width at half maximum empirically determined to $60 \mathrm{~km}$ ), separately.

\section{ZENITH TOTAL DELAY FROM THE SBLM}

Profiles with the 4-hour deterministic forecasts of precipitable water vapor, temperature, and pressure for the area of the Envisat track were downloaded from the ECMWF for each of the days. Furthermore, a DEM of the area was provided by the USGS National Elevation Database. The DEM had a horizontal and vertical resolution of 1 arcsecond and $15 \mathrm{~m}$, respectively. However, the DEM was down sampled to 2 arcseconds in order to save computational speed when using the SBLM.

The ECMWF data were interpolated each day of the Envisat overpass using the SBLM. The model assumes that the water vapor and temperature profiles are unchanged above the boundary layer. Within the boundary layer, the shape of the profiles are also unchanged and this is accomplished by stretching the profiles to fit the topography and then combine the stretched profiles within the boundary layer with the original profiles above the boundary layer. The total amount of water vapor, TPW, is then obtained by integration of the topography corrected water vapor profile.

The TPW is nearly proportional to the Zenith Wet Delay (ZWD) with the parameter $\xi$ [13], which is a weak function of the weighted mean temperature of the atmosphere column, $T_{m}$, according to

$$
\xi=\frac{10^{6}}{\left(\rho R_{v}\left(k_{2}^{\prime}+\frac{k_{3}}{T_{m}}\right)\right)}
$$

Here $k_{2}^{\prime}$ and $k_{3}$ are empirical physical constants, $\rho$ is the density of liquid water and $R_{v}$ is the specific gas constant for water vapor. $T_{m}$ can be approximated using the surface temperature, $T$, from the empirical relation [13]

$$
T_{m}=0.72 T+70.2
$$

Furthermore, in order to obtain the ZTD, the Zenith Hydrostatic Delay (ZHD) has to be estimated and this can be done according to [14]

$$
Z H D=\frac{0.22765 P}{1-0.00266 \cos 2 \lambda-0.00028 h}
$$

where $P$ is surface pressure in mbar, $\lambda$ is the latitude, $h$ is the geodetic height in $\mathrm{km}$, and ZHD is given in $\mathrm{cm}$.

From Eq. 1, Eq. 2, and Eq. 3 the TPW maps from the SBLM were converted into ZTD maps for each day using surface temperature and pressure from the SBLM.

\section{TROPOSPHERIC CORRECTION MAPS}

The ZTD maps for each day (from both GPS and the SBLM) were then converted into ZTD correction maps (difference between the two days) and combined ZTD difference maps were created. In order to gain from both the high spatial resolution of the SBLM ZTD maps and the high precision of the GPS ZTD observations the two data sets were combined so that the SBLM ZTD field agreed with the GPS ZTD observations at the sites of the GPS stations. Thereafter, the difference was interpolated using IDW and Gaussian interpolation, separately, and added to the SBLM ZTD surface. 


\section{RESULTS}

The resulting correction maps (GPS, SBLM and the combinations from both IDW and Gaussian interpolation) were applied to the three InSAR images. Since the temporal baselines of the InSAR images were short, no surface deformation should be visible (except for perhaps the third InSAR image with a 105 days temporal baseline), meaning that what is seen in the InSAR images should be the difference in troposphere. Therefore, after applying the tropospheric correction maps, the resulting image should be residuals of difference in troposphere.

The Root-Mean-Square (RMS) errors of the each InSAR image was calculated both before and after applying the tropospheric corrections. A reduction of the RMS errors of 15$32 \%$ was seen for two of the InSAR images, whereas the RMS error for the third image increased with $3-8 \%$. In both shortinterval InSAR images gradients of different magnitudes were seen, indicating residual orbit errors [15]. After promising results estimating the orbit errors with plane fitting [16], the tropospheric corrected InSAR images were reprocessed with ROI_pac, estimating new orbits using a quadratic fit. The RMS error of the reprocessed InSAR images (after first applying the tropospheric correction) decreased with $15-68 \%$ compared to the RMS error of the original InSAR images. As an example of the tropospheric correction, Fig. 1 shows one of the original InSAR images (July-August), a GPS correction map, and the corrected InSAR image (both troposphere and orbit corrected).

\section{CONCLUSIONS AND OUTLOOK}

In this report, profiles from the European Centre for MediumRange Weather Forecasts (ECMWF) and Zenith Total Delay from the Global Positioning System (GPS) were used to produce tropospheric correction maps for Interferometric Synthetic Aperture Radar (InSAR). The ECMWF data were interpolated into a finer grid with the Stretched Boundary Layer Model (SBLM), utilizing the high horizontal resolution of a Digital Elevation Model (DEM), and converted into ZTD maps. The GPS ZTD data were interpolated using inverse distance weighted and Gaussian interpolation. Furthermore, a combined SBLM and GPS correction product was developed.

When evaluating the tropospheric correction maps on three Envisat InSAR images, with short temporal baselines, the RMS was greatly reduced with an RMS reduction of up to $32 \%$. However, for one of the images the RMS increased and large errors remained after applying the tropospheric correction. Furthermore, the residuals showed a constant gradient over the area for two of the images, suggesting a remaining orbit error. The tropospheric corrected InSAR images were reprocessed which resulted in the overall RMS reduction of $15-68 \%$.

By correcting for the troposphere, other errors become visible. The main contributor to the remaining errors is uncertainties with determining the satellite orbit. Because the orbit error is now separated from the tropospheric error, the orbit can be corrected for more accurately. To conclude, the tropospheric correction enables orbit correction and by correcting for both errors, the quality for the InSAR images increased significantly.

The results show that it is possible to reduce tropospheric errors in InSAR images using available data sets together with simplistic algorithms. Future work consists of further developing the combination of the ZTD from GPS and weather models and implementing it into the GPS Explorer portal [17] in the project Real-Time In Situ Measurements for Earthquake Early Warning and Spaceborne Deformation Measurement Mission Support. In this project the aim is to provide tropospheric correction maps operationally through a web portal. Additionally, there is today ongoing work of studying algorithms for elevation-based interpolation of weather model data with the AIST project Online Services for Correcting Atmosphere in Radar.

\section{ACKNOWLEDGEMENTS}

This research was carried out at the Jet Propulsion Laboratory, California Institute of Technology, under a contract with the National Aeronautics and Space Administration. Data provided by the European Space Agency through WInSAR.

We thank Stephanie L. Granger and Susan E. Owen for helpful discussions.

\section{REFERENCES}

[1] P. A. Rosen, S. Hensely, I. R. Joughin, F. K. Li, S. N. Madsen, E. Rodriguez, and R. M. Goldstein, "Synthetic Aperture Radar Interferometry," Proceedings of the IEEE, vol. 88, no. 3, pp. 333-382, 2000.

[2] D. Massonnet, K. Feigl, M. Rossi, and F. Adragna, "Radar interferometric mapping of deformation in the year after the Landers earthquake," Nature, vol. 369, pp. 227$230,1994$.

[3] H. A. Zebker, P. A. Rosen, and S. Hensley, "Atmospheric effects in interferometric synthetic aperture radar surface deformation and topographic maps," J. Geophys. Res., vol. 102(B4), pp. 7547-7563, 1997.

[4] S. Williams, Y. Bock, and P. Fang, "Integrated satellite interferometry: Tropospheric noise, GPS estimates and implications for interferometric synthetic aperture radar products," J. Geophys. Res., vol 103(B11), pp. 27051-27068, 1998.

[5] Z. Li, E. Fielding, P. Cross, and J.-P. Muller, 'Interferometric synthetic aperture radar atmospheric correction: 

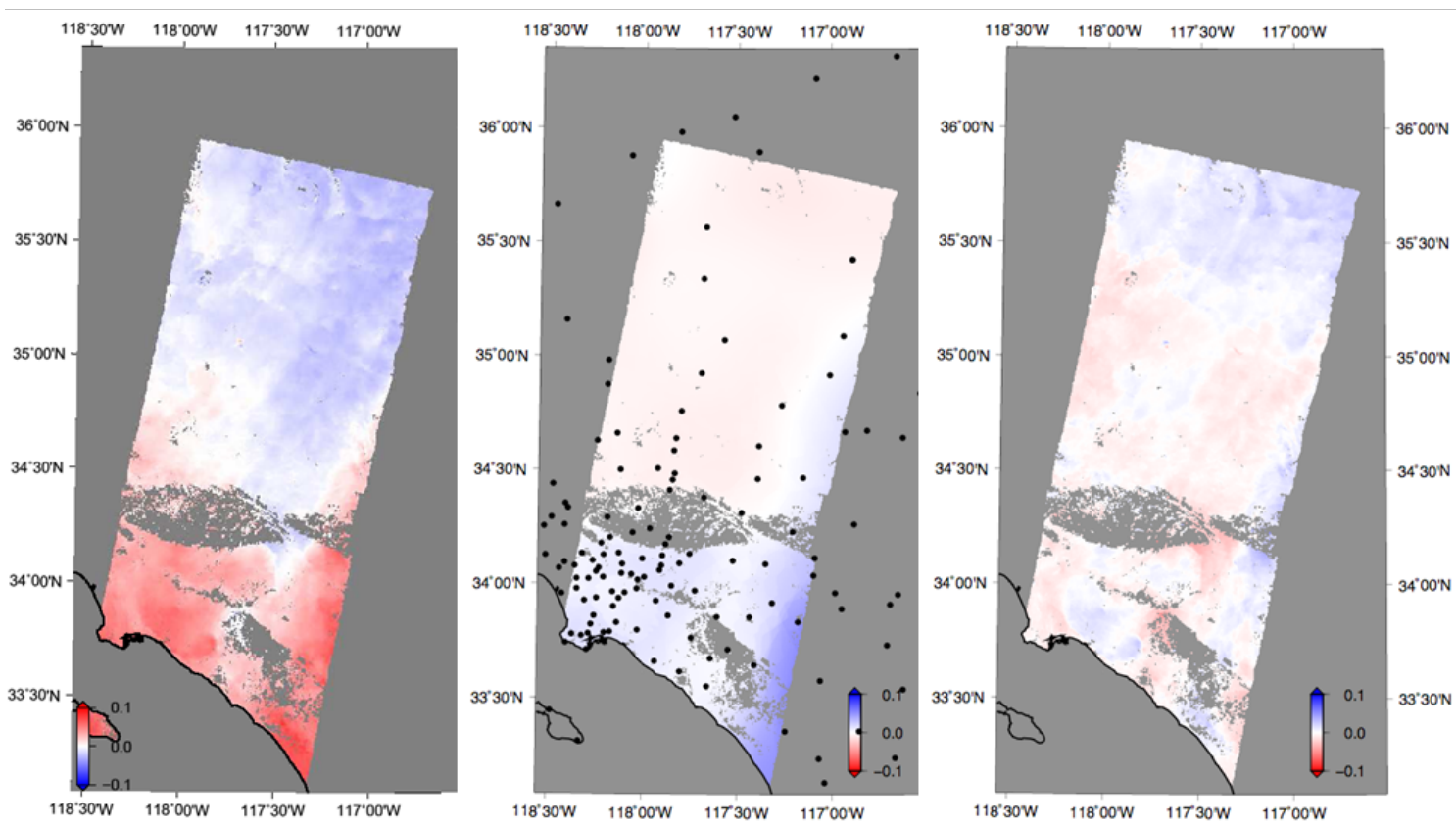

Fig. 1. The original July-August 2006 InSAR image (left), the GPS ZTD correction map (center), and the troposphere and orbit corrected InSAR image (right) with values in $\mathrm{m}$. The black dots in the correction map indicates the location of the GPS stations and this map is applied additively to the original InSAR image. Envisat data copyright 2006 ESA.

GPS topography-dependent turbulence model," J. Geophys. Res., vol. 111, B02404, 12 pp, 2006.

[6] F. Onn and H. A. Zebker, "Correction for interferometric synthetic aperture radar atmospheric phase artifacts using time series of zenith wet delay observations from a GPS network," J. Geophys. Res., vol. 111, B09102, 16 pp, 2006.

[7] P. A. Rosen, S. Hensley, G. Peltzer, and M. Simons, "Updated Repeat Orbit Interferometry package released," Eos Trans. AGU, vol. 85, no. 5, 47 pp, 2004.

[8] C. W. Chen and H. A. Zebker, "Network approaches to two-dimensional phase unwrapping: intractability and two new algorithms," J. Opt. Soc. Am. A, vol. 17, pp. 401-414, 2000 .

[9] D. Massonnet, K. L. Feigl, H. Vadon, and M. Rossi, "Coseismic deformation field of the $\mathrm{M}=6.7$ Northridge, California earthquake of January 17, 1994 recorded by two radar satellites using interferometry," Geophys. Res. Lett., vol. 23, no. 9, pp. 969-972, 1996.

[10] F. H. Webb, and J. F. Zumberge, "An introduction to the GIPSY/OASIS-II," JPL Publ., D-11088, 1993.

[11] J. F. Zumberge, M. B. Heflin, D. C. Jefferson, M. M. Watkins, and F. H. Webb, "Precise point positioning for the efficient and robust analysis of GPS data from large networks," J. Geophys. Res., vol. 102, pp. 5005-5017, 1997.
[12] J. Boehm, A. Niell, P. Tregoning, and H. Schuh, "Global Mapping Function (GMF): A new empirical mapping function based on numerical weather model data," Geophys. Res. Lett., vol. 33, L07304, 2006.

[13] M. Bevis, S. Businger, S. Chiswell, T. A. Herring, R. A. Anthes, C. Rocken, and R. H. Ware, "GPS meteorology: mapping zenith wet delays onto precipitable water," $J$. Appl. Meteorol., vol. 33(3), pp. 379-386, 1994.

[14] J. Saastamoinen, "Atmospheric correction for the troposphere and stratosphere in radio ranging for satellites," in The Use of Artificial Satellites for Geodesy, Geophys. Monogr. Ser., vol. 15, edited by S. W. Henriksen et al., pp. 247-251, AGU, Washington D.C., 1971.

[15] A. O. Kohlhase, K. L. Feigl, and D. Massonnet, "Applying differential InSAR to orbital dynamics: a new approach for estimating ERS trajectories," J. Geodesy, vol. 77, pp. 493-502, 2003.

[16] D. Massonnet and K. Feigl, "Radar interferometry and its applications to changes in the Earths surface," Rev. Geophys., vol. 36, no. 4, pp. 441-500, 1998.

[17] F. Webb, Y. Bock, S. Kedar, S. E. Owen, D. Dong, P. Jamason, P. Fang, M. B. Squibb, B. W. Crowell, and D. Avraham, "Solid Earth Science Data System for Exploration of Lithospheric Deformation in the Western US," Eos. Trans. $A G U$, vol. 90, Fall Meet. Suppl., Abstract IN42A-07, 2009. 